\title{
Study on Status and Willingness towards Hepatitis B Vaccination among Migrant Workers in Chongqing, China: A Cross-Sectional Study
}

\author{
Hui Xiang ${ }^{1,2,3}$, Xiaojun Tang ${ }^{1,2,3}$, Meng Xiao ${ }^{1,2,3}$, Lin Gan ${ }^{1,2,3}$, Kun Chu ${ }^{1,2,3}$, Shan Li ${ }^{4}$, \\ Yu Tian ${ }^{5}$ and Xun Lei ${ }^{1,2,3, *}$ \\ 1 School of Public Health and Management, Chongqing Medical University, Chongqing 400016, China; \\ ashely1010@163.com (H.X.); 100108@cqmu.edu.cn (X.T.); felicity_xiao@163.com (M.X.); \\ qq1414154380@163.com (L.G.); chukun@stu.cqmu.edu.cn (K.C.) \\ 2 Research Center for Medicine and Social Development, Chongqing Medical University, \\ Chongqing 400016, China \\ 3 Innovation Center for Social Risk Governance in Health, Chongqing Medical University, \\ Chongqing 400016, China \\ 4 School of Clinical Medicine, Chongqing Medical University, Chongqing 400016, China; \\ antonio19960424@icloud.com \\ 5 Nan'an District Center for Disease Control and Prevention, Chongqing 400067; nacdcty@163.com \\ * Correspondence: leixun521@163.com
}

Received: 23 August 2019; Accepted: 21 October 2019; Published: 22 October 2019

\begin{abstract}
Background: Rural-to-urban migrant workers may serve as a bridge population for the cross-regional spread of hepatitis B vaccination (HBV) due to frequent shifts between their work areas and homelands, and they are less likely to be covered by the national hepatitis B (HB) immunization program. This study aimed to investigate the current inoculation status of HB vaccine among migrant workers and the willingness to be vaccinated among non-vaccinated ones. Methods: We conducted a cross-sectional survey using anonymous interviews with migrant workers selected by two-stage cluster sampling from July to December 2018. Binary logistic regression models were adopted to detect influencing factors associated with $\mathrm{HB}$ inoculation status and vaccination willingness. Results: 1574 respondents were recruited in the surveys, and $773(49.11 \%)$ respondents reported that they had been inoculated with $\mathrm{HB}$ vaccine. Only 285 (35.58\%) non-vaccinated respondents were willing to be inoculated. Logistic regression indicated that younger age, higher education level, less wearing of condoms, higher knowledge scores of $\mathrm{HB}$, and higher risk perception of $\mathrm{HBV}$ infection were positively associated with inoculation of $\mathrm{HB}$ vaccine. Respondents who were more highly educated, and drinkers, with higher knowledge scores of $\mathrm{HB}$ and with higher risk perception of HBV infection were more willing to be vaccinated. Conclusions: the $\mathrm{HB}$ vaccination rate of migrant workers in Chongqing was relatively low and only a small section of non-vaccinated migrant workers had vaccination willingness. Health interventions and policies are needed to improve knowledge and cognition of HB among migrant workers, particularly for those who are older, less educated, poor in HB knowledge, less likely to wear condoms, and non-drinkers. Peer education, as well as the combination of traditional and new media, would be accessible and effective ways to disseminate $\mathrm{HB}$ related knowledge for migrant workers.
\end{abstract}

Keywords: migrant workers; hepatitis B; vaccination status; willingness to vaccinate

\section{Introduction}

Hepatitis B (HB) remains a challenge to public health, causing 500,000 to 750,000 deaths per year due to cirrhosis and liver cancer evolving from HB worldwide [1]. Currently, World Health 
Organization (WHO) urges countries to invest in eliminating hepatitis once again, and underlined that the low- and middle-countries are still the main battlefields [2]. China is one of the countries with high endemicity of $\mathrm{HB}$ reported by the WHO [3], with an estimated number of 93 million HB patients and 100,000 annually new hepatitis B virus (HBV) infections [4]. According to the National Hepatitis Seroepidemiology Survey in 1992 and 2006, the positive rate of hepatitis B surface antigen (HBsAg) of Chinese aged between 1 and 59 decreased from $9.75 \%$ to $7.18 \%$ [5]. However, the overall HBsAg positive rate was still over $8 \%$ for Chinese adults in 2016 [5].

$\mathrm{HB}$ vaccination has been proved to be the most efficient and economical way to prevent HBV infection and spread [6]. Previous study highlighted that HB vaccine could provide long-term protection against risks of infection encountered in later life [7]. The WHO advocated to enlarge the coverage of HB vaccination prior to 1991 [8], and the Chinese Ministry of Health (MoH) began to offer free HB immunization for neonates after 2002 with free supply being expanded to children under the age of 15 after 2009 [9]. Because of this, the HBsAg positive rate of children under five decreased to $0.32 \%$ in 2014 [10], and the reduction of 90\% met the WHO's goals of which the WHO's Western Pacific Regional Committee had passed a resolution in 2005 to reduce HBV prevalence in children to less than $1 \%$ by 2017 [11]. However, China's adults were not covered by the free immunization program due to the limited health resource [9].A national survey showed that the HBsAg positive rate in populations aged 15 to 59 was $8.75 \%$ in 2006 [5].

With rapid development and urbanization, a large number of young adults have migrated from rural areas to big cities for better employment opportunities and higher incomes in China [12]. According to the latest Migrant Workers Monitoring and Investigation Report of China, the number of migrant workers reached up to 288.36 million in 2018 [13]. Migrant workers basically have low education levels and poor self-protected awareness, and most of them live a stressful life and lack of access to health care, where they seem to have a higher likelihood of HBV infection than non-migrant workers $[14,15]$. The frequent travels make migrant workers a possible bridge population for HBV transmission [16].

A previous study found that the HBsAg positive rates of migrant workers $(8.8 \%)$ and rural residents $(9.1 \%)$ were both higher than other groups such as students $(7.1 \%)$, workers $(5.9 \%)$, cadres (4.4\%), teachers $(6.4 \%)$, and health care workers ( $4.9 \%)$ in northwestern China [17]. Likewise, HBsAg positive rate of migrant workers $(7.05 \%)$ is higher than that of local residents $(6.32 \%)$ in Shenzhen city [18]. The Action Plan for Prevention and Treatment of Viral Hepatitis in China (2017-2020) underlined the need to explore the vaccination strategy and improve HB inoculation for the susceptible and high-risk population [19].

Chongqing is one of the newly promoted first-line metropolises of China where immigration has gradually grown over the years. Nevertheless, studies focused on the HB immunization status among Chongqing's migrant workers have been rarely seen so far. The present study aimed to investigate the current situation of $\mathrm{HB}$ vaccination and vaccinating willingness among migrant workers and detect the potential influencing factors to provide evidence for recommendation and exploration of scale-up immunization programs targeting migrants in China.

\section{Methods}

\subsection{Study Sites and Participants}

The present study was performed in Chongqing, the largest municipality directly under the Chinese central government. Chongqing, located in southwestern China, is referred to as a "miniature of China" because its geographic characteristics, social-economic profile, and urban-rural distribution are close to the national average [20]. The city area of Chongqing, one of the busiest regions for the inflow of China's migrant population, consists of nine administrative districts with 8.65 million residents, among which immigrants account for 23.5\% [21]. In 2007, the estimated HBsAg positive rate 
was $8.6 \%$ among migrant workers in the city area of Chongqing [22]. According to the Health Statistic Yearbook of Chongqing, there were nearly 26,000 new infections of viral hepatitis in 2016 [23].

The target subjects in the study were migrant workers who (1) were 18 years and above, (2) had been in the city area of Chongqing for at least six months, (3) had not registered as Chongqing urban resident, (4) were engaged mainly in the secondary or tertiary industry, such as construction industry, manufacturing industry, wholesale and retail industry, transportation industry, hotel and catering industry, community services. Individuals who were not able to understand the questionnaire items or refused to be surveyed were excluded.

\subsection{Sampling Methods}

Field surveys were carried out from July to December 2018. Participants were selected by two-stage stratified cluster sampling-first, nine districts of the city area in Chongqing were categorized into three stratifications (very developed, medium developed, and less developed) according to economic background, geographic location and population density, and three districts were then randomly selected from each stratification respectively. Second, two or more enterprise units were purposefully selected within each district, including the manufacturing, construction, wholesale and retail industry, the transportation industry, the hotel and catering industry, community services, with the assistance of local Center for Disease Control and Prevention, Health supervision Institute, and Urban-rural Development Committee. All migrant workers meeting the inclusion criteria were sampled from each enterprise [13].

\subsection{Study Instruments}

The questionnaire was constructed based on the WHO fact sheet on HB immunization and the questionnaires adopted in the published studies on knowledge related to HB epidemics and prevention [24-26]. A pilot test was performed with 100 migrant workers in restaurants near to Chongqing Medical University (CQMU) and the framework and wordings of the questionnaire were modified for better understanding of target subjects. The final version consisted of four modules including socio-demographics (16 items), status, and willingness of vaccination (three items), knowledge of HB (14 items), perceived and behavioral risks (eight items). Detailed items and scales of the questionnaire can be found in the Supplementary Materials.

\subsection{Statistical Analysis}

Survey data were double-checked and entered by Epidata 3.1 (The EpiData Association, Odense, Denmark). All data were analyzed using SAS software, version 9.4 (SAS Institute, Cary, North Carolina, USA). Categorical variables were described by number and percentage. Continuous variables were described by the mean and standard deviation (normally distributed variables) or the median and interquartile range (abnormally distributed). Then, univariate analyses were performed using the Chi-square test or Wilcoxon rank sum test to assess the HB vaccination status and willingness of the respondents with variables in the modules of social demographics, knowledge of hepatitis $B$, and perceived and behavioral risk. Variables with $P$-values less than 0.10 were subsequently inputted into the binary logistic regression model to test the possible influencing factors for two outcomes-(1) HB vaccination status and (2) vaccinating willingness. Adjusted odds ratios (ORs) and $95 \%$ confidence intervals (CIs) of variables were computed and the test level was $\alpha=0.05, \beta=0.10$.

\subsection{Ethics Statement}

The present study was approved by the Institutional Review Board of CQMU (2018016). Participants were reassured that all responses would be anonymous and written informed consent was secured from each respondent. Peak working hours were avoided to ensure the quality of interviews. 


\section{Results}

A total of 1740 participants met the eligible criteria and 1574 (90.46\%) repondents completed this survey. The median age was $32.06 \pm 21.18$ years, ranging from 18 to 68 while 1487 (94.47\%) of respondents were ethnic Han and 87 (5.53\%) respondents were ethnic minority. A number of 223 $(14.17 \%)$ respondents had an education background of primary school or below, and 579 (36.79\%) respondents had an education background of junior middle school. $561(35.64 \%)$ respondents had a personal monthly income between 2500 and $4000 \mathrm{RMB}$, while 364 (23.13\%) respondents earned less than 2500 RMB a month (Table 1).

A number of 801 (50.89\%) respondents reported that they had not inoculated HB vaccine, among whom there were $516(64.42 \%)$ unwilling to vaccinate. The 745 (42.01\%) non-vaccinated respondents were mainly aged between 18 and 30, among whom there were only 285 (35.58\%) willing to be vaccinated in the future (Table 2). To explore the reason for not having HB vaccination, 206 (40\%) respondents said that they had never heard of HB vaccination, 160 (31.11\%) respondents did not know where to vaccinate, $84(16.30 \%)$ respondents doubted the effectiveness or safety of HB vaccine, $55(10.74 \%)$ respondents thought vaccination was inconvenient or inaccessible, and $55(10.74 \%)$ respondents had followed their friends or family members in not vaccinating (Table 3).

The HB knowledge score was $5.00 \pm 6.00$ and $7.00 \pm 3.00$ among respondents in non-vaccinated and vaccinated respondents respectively (Table 1). The most popular access for the respondents to obtain HB knowledge and information is hearing from friends or family members (53.88\%), followed by television or radio (38.12\%), Internet or mobile phone $(27.95 \%)$, and newspapers or magazine $(19.70 \%)$ respectively (Table 4$)$.

A number of $678(43.07 \%)$ respondents thought that it was absolutely impossible/impossible for them to get HB infection. In the last six months, 184 (11.69\%) respondents had had casual sex, 92 $(5.84 \%)$ of them had commercial sex, and $52(3.30 \%)$ respondents had homosexual behaviors or anal sex activities. There were 688 (43.71\%) respondents who had had sex with a condom less than five times in the past half a year. In addition, 17 (1.08\%) respondents had intravenous drug use by sharing injectors, 23 (1.46\%) respondents had experienced illegal blood selling or transfusion, and $298(18.93 \%)$ respondents had shared toothbrush or towels with others in the last six months (Table 1).

All continuous variables were abnormally distributed. As Table 1 shows, univariate analyses using $\chi^{2}$ test or Wilcoxon sum rank test indicated that HB vaccination status of migrant workers was significantly different with variables of ages, hometown, ethnicity, education background, years of being a migrant worker, job position, working hours per day, monthly personal income, cigarette consumption, knowledge score of $\mathrm{HB}$, perceived risk to $\mathrm{HB}$, commercial sex behavior, and condom use respectively $(P<0.05)$. Binary logistic regression showed that higher education level $(\mathrm{OR}=1.85,95 \%$ CI: $1.24-2.74 ; \mathrm{OR}=1.94,95 \% \mathrm{CI}: 1.25-3.00 ; \mathrm{OR}=2.58,95 \% \mathrm{CI}: 1.57-4.24)$, higher knowledge scores (OR $=1.17,95 \% \mathrm{CI}: 1.13-1.22)$, higher risk perception of $\mathrm{HB}(\mathrm{OR}=1.40,95 \% \mathrm{CI}: 1.11-1.75)$ and less condom usage $(\mathrm{OR}=1.35,95 \% \mathrm{CI}: 1.07-1.70)$ were associated with having been vaccinated. While age was negatively associated with $\mathrm{HB}$ vaccination among migrant workers (OR = 0.98, 95\% CI: 0.96-0.99).

As Table 2 shows, univariate analyses using $\chi^{2}$ test or Wilcoxon sum rank test indicated that the willingness of $\mathrm{HB}$ vaccination for non-vaccinated respondents was significantly different with variables of gender, ages, ethnicity, education background, years of being a migrant worker, working hours per day, sending money to family, knowledge score of $\mathrm{HB}$, perceived risk of $\mathrm{HB}$, and condom use $(P<0.05)$. Binary logistic regression showed that migrant workers with higher education level $(\mathrm{OR}=2.01,95 \% \mathrm{CI}: 1.24-3.28 ; \mathrm{OR}=2.21,95 \% \mathrm{CI}: 1.26-3.89)$, drinking $(\mathrm{OR}=1.84,95 \% \mathrm{CI}: 1.23-2.76)$, with better knowledge scores $(\mathrm{OR}=1.13,95 \% \mathrm{CI}: 1.08-1.19)$ and higher risk perceptions of HB (OR $=2.18,95 \%$ CI:1.57-3.03) were more willing to be inoculated. Respondents of ethnic minority were unwilling to vaccinate compared with those of ethnic Han (OR = 0.46, 95\% CI: 0.22-0.95). 
Table 1. Univariate analysis and binary logistic regression on factors associated with vaccination status in hepatitis B vaccination (HBV) vaccine for migrant workers ( $\mathrm{N}=1574)$.

\begin{tabular}{|c|c|c|c|c|c|c|c|}
\hline \multirow[b]{2}{*}{ Variables } & \multirow[b]{2}{*}{ Total } & \multicolumn{4}{|c|}{ Univariate Analysis } & \multicolumn{2}{|c|}{ Binary Logistic Regression } \\
\hline & & $\begin{array}{c}\text { Non-Vaccinated } \\
(\mathrm{N}=801)\end{array}$ & $\begin{array}{l}\text { Vaccinated } \\
(\mathrm{N}=773)\end{array}$ & $x^{2} / Z^{a}$ & $P^{\mathrm{b}}$ & $\mathrm{OR}^{\mathrm{c}}$ & $95 \% \mathrm{CI}^{\mathrm{d}}$ \\
\hline \multicolumn{8}{|l|}{ Gender } \\
\hline Male & 781 & $412(52.75)$ & $369(47.25)$ & 2.15 & 0.142 & & \\
\hline Female & 793 & $389(49.05)$ & $404(50.95)$ & & & & \\
\hline Age & & $39.42 \pm 23.94$ & $29.53 \pm 14.85$ & -8.50 & $<0.001$ & 0.98 & $(0.96,0.99)$ \\
\hline \multicolumn{8}{|l|}{ Hometown } \\
\hline Rural area in Chongqing & 1121 & $593(52.90)$ & $528(47.10)$ & 6.29 & 0.012 & Ref & \\
\hline Rural area in other cities/provinces & 453 & $208(45.92)$ & $245(54.08)$ & & & 1.19 & $(0.94,1.52)$ \\
\hline \multicolumn{8}{|l|}{ Ethnicity } \\
\hline & 1487 & $747(50.24)$ & $740(49.76)$ & 4.61 & 0.032 & Ref & \\
\hline Minority & 87 & $54(62.07)$ & $33(37.93)$ & & & 0.66 & $(0.40,1.08)$ \\
\hline \multicolumn{8}{|l|}{ Education background } \\
\hline Primary school or below & 223 & $173(77.58)$ & $50(22.42)$ & 112.99 & $<0.001$ & Ref & \\
\hline Junior middle school & 579 & $318(54.92)$ & $261(45.08)$ & & & 1.85 & $(1.24,2.74)$ \\
\hline High school & 524 & $231(44.08)$ & $293(55.92)$ & & & 1.94 & $(1.25,3.00)$ \\
\hline College and above & 248 & $79(31.85)$ & $169(68.15)$ & & & 2.58 & $(1.57,4.24)$ \\
\hline \multicolumn{8}{|l|}{ Marital status } \\
\hline Single & 367 & $182(49.59)$ & $185(50.41)$ & 1.74 & 0.419 & & \\
\hline Married/Having a relationship & 1142 & $581(50.88)$ & $561(49.12)$ & & & & \\
\hline Divorced/Widowed & 65 & $38(58.46)$ & $27(41.54)$ & & & & \\
\hline \multicolumn{8}{|l|}{ Live with the spouse/partner } \\
\hline No & 778 & $391(50.26)$ & $387(49.74)$ & 0.25 & 0.620 & & \\
\hline Yes & 796 & $410(51.51)$ & $386(48.49)$ & & & & \\
\hline \multicolumn{8}{|l|}{ Accommodation } \\
\hline Self-renting room/Self-purchased house & 861 & $429(49.83)$ & $432(50.17)$ & 0.86 & 0.354 & & \\
\hline Co-renting room/Dormitory & 713 & $372(52.17)$ & $341(47.83)$ & & & & \\
\hline Years of being a migrant worker & & $13.00 \pm 10.92$ & $5.00 \pm 8.00$ & -4.55 & $<0.0001$ & 0.98 & $(0.97,1.00)$ \\
\hline \multicolumn{8}{|l|}{ Type of work } \\
\hline Secondary industry ${ }^{\mathrm{e}}$ & 851 & $428(50.29)$ & $423(49.71)$ & 0.26 & 0.608 & & \\
\hline Tertiary Industry ${ }^{f}$ & 723 & $373(51.59)$ & $350(48.41)$ & & & & \\
\hline \multicolumn{8}{|l|}{ Job Position } \\
\hline Ordinary employee & 1293 & $694(53.67)$ & $599(46.33)$ & 22.46 & $<0.001$ & Ref & \\
\hline Team/Administrator & 281 & $107(38.08)$ & $174(61.92)$ & & & 1.07 & $(0.82,1.40)$ \\
\hline Working hours per day & & $9.00 \pm 4.00$ & $10.00 \pm 4.00$ & 2.85 & 0.004 & 1.04 & $(0.98,1.10)$ \\
\hline \multicolumn{8}{|l|}{ Monthly personal income (RMB) } \\
\hline$<2500$ & 364 & $194(53.30)$ & $170(46.70)$ & 6.19 & 0.045 & Ref & \\
\hline 2501 4000 & 561 & $301(53.65)$ & $260(46.35)$ & & & 0.79 & $(0.57,1.09)$ \\
\hline$>4000$ & 649 & $306(47.15)$ & $343(52.85)$ & & & 1.07 & $(0.75,1.53)$ \\
\hline
\end{tabular}


Table 1. Cont.

\begin{tabular}{|c|c|c|c|c|c|c|c|}
\hline \multirow{2}{*}{ Variables } & \multirow{2}{*}{ Total } & \multicolumn{4}{|c|}{ Univariate Analysis } & \multicolumn{2}{|c|}{ Binary Logistic Regression } \\
\hline & & $\begin{array}{l}\text { Non-Vaccinated } \\
(\mathrm{N}=801)\end{array}$ & $\begin{array}{l}\text { Vaccinated } \\
(\mathrm{N}=773)\end{array}$ & $x^{2} / Z^{a}$ & $P^{\mathrm{b}}$ & $\mathrm{OR}^{\mathrm{c}}$ & $95 \% \mathrm{CI}^{\mathrm{d}}$ \\
\hline \multicolumn{8}{|c|}{ Do you regularly send money to your family? } \\
\hline No & 440 & $217(49.32)$ & $223(50.68)$ & 0.60 & 0.437 & & \\
\hline Yes & 1134 & $584(51.50)$ & $550(48.50)$ & & & & \\
\hline \multicolumn{8}{|l|}{ Cigarette consumption } \\
\hline No & 1131 & $550(48.63)$ & $581(51.37)$ & 8.21 & 0.004 & Ref & \\
\hline Yes & 443 & $251(56.66)$ & $192(43.34)$ & & & 0.96 & $(0.74,1.24)$ \\
\hline \multicolumn{8}{|l|}{ Alcohol consumption } \\
\hline No & 1208 & $613(50.75)$ & $595(49.25)$ & 0.04 & 0.835 & & \\
\hline Yes & 366 & $188(51.37)$ & $178(48.63)$ & & & & \\
\hline Knowledge score of HB & & $5.00 \pm 6.00$ & $7.00 \pm 3.00$ & 11.25 & $<0.001$ & 1.17 & $(1.13,1.22)$ \\
\hline \multicolumn{8}{|c|}{ Do you think you are susceptible to HB? } \\
\hline Absolutely impossible/Impossible & 678 & $362(53.39)$ & $316(46.61)$ & 9.17 & 0.010 & Ref & \\
\hline Neutral $\mathrm{T}$ ( & 121 & $72(59.50)$ & $49(40.50)$ & & & 1.04 & $(0.68,1.60)$ \\
\hline Absolutely possible/Possible & 775 & $367(47.35)$ & $408(52.65)$ & & & 1.40 & $(1.11,1.75)$ \\
\hline \multicolumn{8}{|c|}{ Did you have casual sex in the last six months? } \\
\hline No & 1390 & $698(50.22)$ & $692(49.78)$ & 2.16 & 0.142 & & \\
\hline Yes & 184 & $103(55.98)$ & $81(44.02)$ & & & & \\
\hline \multicolumn{8}{|c|}{ Did you have commercial sex in the last six months? } \\
\hline No & 1482 & $745(50.27)$ & 737 (49.73) & 3.89 & 0.049 & Ref & \\
\hline Yes & 92 & $56(60.87)$ & $36(39.13)$ & & & 0.86 & $(0.53,1.40)$ \\
\hline \multicolumn{8}{|c|}{$\begin{array}{l}\text { Did you have homosexual behavior or anal sex activities in } \\
\text { the last six months? }\end{array}$} \\
\hline No & 1522 & $772(50.72)$ & $750(49.28)$ & 0.51 & 0.474 & & \\
\hline Yes & 52 & $29(55.77)$ & $23(44.23)$ & & & & \\
\hline \multicolumn{8}{|c|}{$\begin{array}{l}\text { Did you use condom less than five times when you had the } \\
\text { above sex behavior in the past six months? }\end{array}$} \\
\hline No & 886 & $500(56.43)$ & $386(43.57)$ & 24.93 & $<0.001$ & Ref & \\
\hline Yes & 688 & $301(43.75)$ & $387(56.25)$ & & & 1.35 & $(1.07,1.70)$ \\
\hline \multicolumn{8}{|c|}{$\begin{array}{l}\text { Did you share an injector for intravenous drug use in the } \\
\text { last six months? }\end{array}$} \\
\hline No & 1557 & $793(50.93)$ & $764(49.07)$ & 0.10 & 0.751 & & \\
\hline Yes & 17 & $8(47.06)$ & $9(52.94)$ & & & & \\
\hline \multicolumn{8}{|c|}{$\begin{array}{l}\text { Did you sell blood or have blood transfusions at illegal } \\
\text { clinics in the last six months? }\end{array}$} \\
\hline No & 1551 & $790(50.93)$ & $761(49.07)$ & 0.09 & 0.767 & & \\
\hline Yes & 23 & $11(47.83)$ & $12(52.17)$ & & & & \\
\hline \multicolumn{8}{|c|}{ Did you share toothbrushes/towels in the last six months? } \\
\hline No & 1276 & $649(50.86)$ & $627(49.14)$ & 0.002 & 0.964 & & \\
\hline Yes & 298 & $152(51.01)$ & $146(48.99)$ & & & & \\
\hline
\end{tabular}

a Chi-square test or Wilcoxon sum rank test; ${ }^{\mathrm{b}} \mathrm{P}$-value; ${ }^{\mathrm{c}}$ Odds ratio; ${ }^{\mathrm{d}} 95 \%$ confidence interval; ${ }^{\mathrm{e}}$ Secondary industry includes manufacturing industry and construction industry; ${ }^{\mathrm{f}}$ Tertiary industry includes wholesale and retail industry, transportation industry, hotel and catering industry, community services. 
Table 2. Univariate analysis and binary logistic regression analysis on factors associated with vaccinate willingness in HBV vaccine for migrant workers ( $\mathrm{N}=801$ ).

\begin{tabular}{|c|c|c|c|c|c|c|c|}
\hline \multirow[b]{2}{*}{ Variables } & \multirow[b]{2}{*}{ Total } & \multicolumn{4}{|c|}{ Univariate Analysis a } & \multicolumn{2}{|c|}{ Binary Logistic Regression } \\
\hline & & $\begin{array}{l}\text { Unwilling to Vaccinate } \\
(\mathrm{N}=516)\end{array}$ & $\begin{array}{l}\text { Willing to Vaccinate } \\
(\mathrm{N}=285)\end{array}$ & $x^{2} / Z$ & $P$ & $\mathrm{OR}^{\mathrm{b}}$ & $95 \%$ CI \\
\hline \multicolumn{8}{|l|}{ Gender } \\
\hline Male & 412 & $280(67.96)$ & $132(32.04)$ & 4.64 & 0.031 & Ref & \\
\hline Female & 389 & $236(60.67)$ & $153(39.33)$ & & & 1.18 & $(0.82,1.69)$ \\
\hline Age & & $40.66 \pm 24.85$ & $32.58 \pm 21.24$ & -4.54 & $<0.001$ & 0.99 & $(0.97,1.01)$ \\
\hline \multicolumn{8}{|l|}{ Hometown } \\
\hline Rural area in Chongqing & 593 & $387(65.26)$ & $206(34.74)$ & 0.71 & 0.401 & & \\
\hline Rural area in other cities/provinces & 208 & $129(62.02)$ & 79 (37.98) & & & & \\
\hline \multicolumn{8}{|l|}{ Ethnicity } \\
\hline Han & 747 & $473(63.32)$ & $274(36.68)$ & 5.84 & 0.016 & Ref & \\
\hline Minority & 54 & $43(79.63)$ & $11(20.37)$ & & & 0.46 & $(0.22,0.95)$ \\
\hline \multicolumn{8}{|l|}{ Education background } \\
\hline Primary school or below & 173 & $139(80.35)$ & $34(19.65)$ & 27.86 & $<0.001$ & Ref & \\
\hline Junior middle school & 318 & $202(63.52)$ & $116(36.48)$ & & & 2.01 & $(1.24,3.28)$ \\
\hline High school & 231 & $130(56.28)$ & $101(43.72)$ & & & 2.21 & $(1.26,3.89)$ \\
\hline College and above & 79 & $45(56.96)$ & $34(43.04)$ & & & 1.98 & $(0.97,4.05)$ \\
\hline \multicolumn{8}{|l|}{ Marital status } \\
\hline Single & 182 & $113(62.09)$ & $69(37.91)$ & 0.63 & 0.731 & & \\
\hline Married/Having a relationship & 581 & $379(65.23)$ & $202(34.77)$ & & & & \\
\hline Divorced/Widowed & 38 & $24(63.16)$ & $14(36.84)$ & & & & \\
\hline \multicolumn{8}{|l|}{ Live with spouse/partner } \\
\hline No & 391 & $241(61.64)$ & $150(38.36)$ & 2.58 & 0.108 & & \\
\hline Yes & 410 & $275(67.07)$ & $135(32.93)$ & & & & \\
\hline \multicolumn{8}{|l|}{ Accommodation } \\
\hline Self-renting room/Self-purchased house & 429 & $367(62.24)$ & $162(37.76)$ & 1.92 & 0.166 & & \\
\hline Co-renting room/Dormitory & 372 & $249(66.94)$ & $123(33.06)$ & & & & \\
\hline Years of being a migrant worker & & $7.09 \pm 12.00$ & $6.00 \pm 8.00$ & -2.61 & 0.009 & 0.97 & $(0.94,1.02)$ \\
\hline \multicolumn{8}{|l|}{ Type of work } \\
\hline Secondary industry ${ }^{c}$ & 428 & $282(65.89)$ & $146(34.11)$ & 0.86 & 0.353 & & \\
\hline Tertiary Industry ${ }^{d}$ & 373 & $234(62.73)$ & $139(37.27)$ & & & & \\
\hline \multicolumn{8}{|l|}{ Job Position } \\
\hline Ordinary employee & 694 & $454(65.42)$ & $240(34.58)$ & 2.26 & 0.133 & & \\
\hline Team/Administrator & 107 & $62(57.94)$ & 45 (42.06) & & & & \\
\hline Working hours per day & & $9.00 \pm 3.00$ & $10.00 \pm 4.00$ & 2.41 & 0.016 & 1.07 & $(0.98,1.17)$ \\
\hline \multicolumn{8}{|l|}{ Monthly personal income (RMB) } \\
\hline$<2500$ & 194 & $132(68.04)$ & $62(31.96)$ & 1.63 & 0.442 & & \\
\hline $2501-4000$ & 301 & $188(62.46)$ & $113(37.54)$ & & & & \\
\hline$>4000$ & 306 & $196(64.05)$ & $110(35.95)$ & & & & \\
\hline
\end{tabular}


Table 2. Cont.

\begin{tabular}{|c|c|c|c|c|c|c|c|}
\hline \multirow[b]{2}{*}{ Variables } & \multirow[b]{2}{*}{ Total } & \multicolumn{4}{|c|}{ Univariate Analysis $^{\text {a }}$} & \multicolumn{2}{|c|}{ Binary Logistic Regression } \\
\hline & & $\begin{array}{l}\text { Unwilling to Vaccinate } \\
(\mathrm{N}=516)\end{array}$ & $\begin{array}{l}\text { Willing to Vaccinate } \\
(\mathrm{N}=285)\end{array}$ & $x^{2} / Z$ & $P$ & $\mathrm{OR}^{\mathrm{b}}$ & $95 \% \mathrm{CI}$ \\
\hline \multicolumn{8}{|c|}{ Do you regularly send money to your family? } \\
\hline No & 217 & 364 (62.33) & $220(37.67)$ & 4.11 & 0.043 & Ref & \\
\hline Yes & 584 & $152(70.05)$ & $65(29.95)$ & & & 0.66 & $(0.45,1.02)$ \\
\hline \multicolumn{8}{|l|}{ Cigarette consumption } \\
\hline No & 550 & $346(62.91)$ & 204 (37.09) & 1.75 & 0.186 & & \\
\hline Yes & 251 & $170(67.73)$ & $81(32.27)$ & & & & \\
\hline \multicolumn{8}{|l|}{ Alcohol consumption } \\
\hline No & 613 & 406 (66.23) & $207(33.77)$ & 3.74 & 0.053 & Ref & \\
\hline Yes & 188 & $110(58.51)$ & $78(41.49)$ & & & 1.84 & $(1.23,2.76)$ \\
\hline Knowledge score of HB & & $4.00 \pm 5.00$ & $6.00 \pm 5.00$ & 5.45 & $<0.001$ & 1.13 & $(1.08,1.19)$ \\
\hline \multicolumn{8}{|c|}{ Do you think you are susceptible to HB? } \\
\hline Absolutely impossible/Impossible & 362 & $256(70.72)$ & $106(29.28)$ & 28.53 & $<0.001$ & Ref & \\
\hline Neutral $\mathrm{T}-\mathrm{T}$ & 72 & $58(80.56)$ & 14 (19.44) & & & 0.64 & $(0.33,1.24)$ \\
\hline Absolutely possible/Possible & 367 & $202(55.04)$ & $165(44.96)$ & & & 2.18 & $(1.57,3.03)$ \\
\hline \multicolumn{8}{|c|}{ Did you have casual sex in the last six months? } \\
\hline No & 698 & $451(64.61)$ & 247 (35.39) & 0.09 & 0.766 & & \\
\hline Yes & 103 & $65(63.11)$ & $38(36.89)$ & & & & \\
\hline \multicolumn{8}{|c|}{ Did you have commercial sex in the last six months? } \\
\hline No & 745 & $479(64.30)$ & $266(35.70)$ & 0.07 & 0.789 & & \\
\hline Yes & 56 & $37(66.07)$ & 19 (33.93) & & & & \\
\hline \multicolumn{8}{|c|}{$\begin{array}{l}\text { Did you have homosexual behavior or anal sex activities in } \\
\text { the last six months? }\end{array}$} \\
\hline No & 772 & 496 (64.25) & 276 (35.75) & 0.27 & 0.603 & & \\
\hline Yes & 29 & $20(68.97)$ & $9(31.03)$ & & & & \\
\hline \multicolumn{8}{|c|}{$\begin{array}{l}\text { Did you use a condom less than five times when you had } \\
\text { the above sex behavior in the past six months? }\end{array}$} \\
\hline No & 500 & $343(68.60)$ & $157(31.40)$ & 10.14 & 0.001 & Ref & \\
\hline Yes & 301 & $173(57.48)$ & $128(42.52)$ & & & 1.24 & $(0.89,1.72)$ \\
\hline \multicolumn{8}{|c|}{$\begin{array}{l}\text { Did you share an injector for intravenous drug use in the } \\
\text { last six months? }\end{array}$} \\
\hline No & 793 & 509 (64.19) & $284(35.81)$ & 1.88 & $0.271^{\mathrm{e}}$ & & \\
\hline Yes & 8 & $7(87.50)$ & $1(12.50)$ & & & & \\
\hline \multicolumn{8}{|c|}{$\begin{array}{l}\text { Did you sell blood or have blood transfusions at illegal } \\
\text { clinics in the last six months? }\end{array}$} \\
\hline No & 790 & 507 (64.18) & $283(35.82)$ & 1.47 & 0.344 & & \\
\hline Yes & 11 & $9(81.82)$ & $2(18.18)$ & & & & \\
\hline \multirow{2}{*}{\multicolumn{8}{|c|}{ Did you share toothbrushes/towels in the last six months? }} \\
\hline No & 649 & $426(65.64)$ & & & & & \\
\hline Yes & 152 & $90(59.21)$ & $62(40.79)$ & & & & \\
\hline
\end{tabular}

a Chi-square test or Wilcoxon sum rank test; ${ }^{\mathrm{b}}$ Odds ratio; ${ }^{\mathrm{c}}$ Secondary industry includes manufacturing industry and construction industry; ${ }^{\mathrm{d}}$ Tertiary industry includes wholesale and retail industry, transportation industry, hotel and catering industry, community services; ${ }^{\mathrm{e}}$ Fisher Exact Test. CI: confidence interval. 
Table 3. The reasons for non-vaccination $(\mathrm{N}=516)$.

\begin{tabular}{lc}
\hline \multicolumn{1}{c}{ Non-Vaccination Reasons } & N (\%) \\
\hline Never heard of HB vaccination & $206(40.00 \%)$ \\
Do not know where to vaccinate & $160(31.11 \%)$ \\
Distrust vaccine's effectiveness or safety & $84(16.30 \%)$ \\
Inconvenient to get access & $55(10.74 \%)$ \\
Friends/family members have not vaccinated & $55(10.74 \%)$ \\
Too much self-payment required & $42(8.15 \%)$ \\
Have been infected with HBV & $12(2.22 \%)$ \\
\hline
\end{tabular}

Table 4. Access to hepatitis B (HB) knowledge $(\mathrm{N}=1299)$.

\begin{tabular}{lc}
\hline \multicolumn{1}{c}{ Source of Obtaining HB Knowledge } & N (\%) \\
\hline Friends or family members & $848(53.88 \%)$ \\
Television or radio & $600(38.12 \%)$ \\
Internet or mobile phone & $440(27.95 \%)$ \\
Newspapers or magazines & $310(19.70 \%)$ \\
Doctors & $304(19.31 \%)$ \\
Brochure or booklets & $296(18.81 \%)$ \\
Advertisement & $172(10.93 \%)$ \\
Health education or professional training & $133(8.45 \%)$ \\
Other & $115(7.31 \%)$ \\
\hline
\end{tabular}

\section{Discussion}

According to the political and economic context, $\mathrm{HB}$ vaccination rates vary across different locations in China. In the present study, $49.11 \%$ of migrant workers in the city area of Chongqing had been inoculated with $\mathrm{HB}$ vaccine, which was higher than those of migrant workers in Beijing (37.05\%), Hebei province $(23.58 \%)$, Heilongjiang province $(21.05 \%)$, Jiangsu province $(21.75 \%)$, Ningxia province $(32.81 \%)$, and Hainan province $(17.09 \%)$, but lower than Shenzhen $(70.21 \%)[9,18]$. In addition, the amount of non-vaccinated migrant workers, as much as $51.89 \%$ of respondents, was very much close to the vaccination rate (51.60\%) reported by Gong et al. in 2006 [22], and $42.01 \%$ of non-vaccination respondents were mainly aged between 18 and 30 in our study. This suggested that in the past decades the implementation of the HB immunization program had been inefficient in the countryside of Chongqing and surrounding area, from where the migrant workers mainly flow into city area of Chongqing. Therefore, more specific and stronger policies and regulations are needed to enhance HB immunization in rural areas where the migrant workers mostly come from.

A negative association was detected between $\mathrm{HB}$ vaccination status and age, which is consistent with the study of Liu et al. [9]. On the one hand, aged respondents in our study might be beyond the required age for free $\mathrm{HB}$ immunization by the $\mathrm{MoH}$. On the other hand, aged respondents might take more care of the health of children than themselves [6,9]. In accordance with the study of Yan et al., our findings showed that the more highly educated respondents had a higher vaccination rate and were more willing to be vaccinated if they had not been vaccinated before [27]. Evidence showed that good educational background leads to good cognition of disease prevention among adults, of which the vaccination status of migrant workers differs over educational deviations [28]. In addition, health education campaigns, carried out by staff associations and health service organizations, are also needed to improve migrant workers' attitude both at the workplace and in the community where they live.

HB knowledge scores were shown to be positively associated with both vaccination status and willingness of respondents, because good understanding of $\mathrm{HB}$ related knowledge probably results in concern of HBV infection and awareness of self-protection towards HB [9]. With regard to the ways of obtaining knowledge, friends or family members were the favorite source for migrant workers, which suggests that health interventions based on peer education might work to a large extent. Moreover, 
$\mathrm{TV}$, newspapers, and magazines were still popular among respondents, and Internet/mobile phones also played an important role in information updates for migrant workers, which indicates that health education could be promoted by a combination of traditional and new media.

Consistent with the study by Xie et al., Ethnic Han was detected to be positively associated with willingness to vaccinate compared with the ethnic minorities [29]. Religious faith and briefs have been mentioned as influencing factors of immunization behaviors in previous studies [30], which could be an interpretation for the significantl difference in $\mathrm{HB}$ vaccination between migrant workers of ethnic Han and other minorities from nearby provinces like Yunnan or Guizhou.

Consistent with previous study, the perceived risk of HBV infection was positively associated with $\mathrm{HB}$ vaccination [9]. It makes sense that the more susceptible respondents perceive the risk of infection with HBV the more active they are to vaccinate [31]. Less condom use in the last six months was negatively associated with $\mathrm{HB}$ vaccination. A possible reason was that the vaccinated respondents actually dare to have unprotected sex [32]. Respondents who were alcohol consumers had a strong HB vaccination willingness. It is generally accepted that long-term drinking might be a potential cause of liver damage. Therefore, drinkers are concerned more about liver protection and are more willing to be inoculated.

Unlike some published studies, income was not detected to significantly affect either HB vaccination or vaccinating willingness as the balanced distributions within every income levels in our study showed [33]. Having said that, $8.15 \%$ of non-vaccinated respondents argued that too much self-payment was a big obstacle. Therefore, more subsidy or reimbursement targeting non-vaccinated adults is necessary to explore to reduce the financial burden of migrant workers [9].

In addition, only $35.58 \%$ of non-vaccinated respondents expressed willingness to vaccinate in the future. The top-three reasons for their missing of $\mathrm{HB}$ vaccination were not having heard of the vaccination, unclear where the vaccination sites were situated, and distrustful of the effectiveness or safety, which suggested that an urgent task of enhancing publicity of HB knowledge and dissemination of health and immunization services should be advanced not only by health education and promotion but also by joint effort and cooperation among the relevant administrative departments.

\section{Limitations of the Study}

Some limitations of the present study should be acknowledged. First, as it was a cross-sectional study, any causal inference should be made cautiously on the basis of the association observed in our study. Second, respondent non-random sampling was adopted due to the limitation of the availability and acceptance of subjects, which may cause a selection bias in the occupational distribution between sampled respondents and the overall migrant workers in Chongqing. Third, data collected by interviews may generate a recall bias both on $\mathrm{HB}$ vaccination status and risk behaviors. Therefore, a longitudinal study with a larger sample size by quota sampling, matching with vaccination records or serological examination, is needed to make the findings more robust.

\section{Conclusions}

Approximately one half of the migrant workers had been inoculated with HB vaccine in the present study. The non-vaccinated respondents were relatively low in their willingness to be vaccinated. Younger age, higher education levels, higher knowledge scores, higher perceived risk of HB, and less condom use were associated with $\mathrm{HB}$ vaccination for migrant workers. Migrant workers who were better educated, drank alcohol, with higher knowledge scores and higher perception of HB susceptibility were more willing to inoculate $\mathrm{HB}$ vaccine. Health education and campaigns related to HB prevention should be focused on non-vaccinated migrant workers who are older, of lower education, and poor in HB knowledge. Peer education works for the circulation of HB knowledge, and new social media like the internet and smart phone apps will play an important role in addition to traditional social media like television, radio, and magazines. 
Supplementary Materials: The following are available online at http:/www.mdpi.com/1660-4601/16/20/4046/s1, Questionnaire for migrant workers.

Author Contributions: X.L. and X.T. conceived and designed the study. X.L., H.X., M.X., L.G., K.C., S.L. performed field investigation and data collection. H.X. and X.L. conducted data analysis. X.L. and H.X. drafted the Abstract with input from X.T. and X.L. reviewed and polished the Abstract. All authors have seen and approved the final version of the Abstract for publication.

Funding: This research was funded by National Natural Science Foundation of China, grant number 71603034.

Acknowledgments: We would like to thank local health institutions for their kind assistance and coordination during the field study. All the migrant workers who participated in the survey are much appreciated.

Conflicts of Interest: The authors declare no conflicts of interest.

\section{References}

1. Howell, J.; Van Gemert, C.; Lemoine, M.; Thursz, M.; Hellard, M. Overview of Hepatitis B Prevalence, Prevention, and Management in the Pacific Islands and Territories. J. Gastroenterol. Hepatol. 2014, 29, 1854-1866. [CrossRef]

2. World Health Organization. Who Urges Countries to Invest in Eliminating Hepatitis. 2019. Available online: https://www.who.int/news-room/detail/26-07-2019-who-urges-countries-to-invest-in-eliminatinghepatitis (accessed on 3 August 2019).

3. Li, T.Y.; Yang, Y.; Zhou, G.; Tu, Z.K. Immune Suppression in Chronic Hepatitis B Infection Associated Liver Disease: A Review. World J. Gastroenterol. 2019, 25, 3527-3537. [CrossRef]

4. Chinese Center for Disease Control and Prevention. The Control of Hepatitis B Has Achieved Remarkable Results in China. 2013. Available online: http://www.nhc.gov.cn/jkj/s3582/201307/ 518216575e544109b2caca07fca3b430.shtml (accessed on 3 August 2019). (In Chinese)

5. Qiu, Y.; Ren, J.; Yao, J. Healthy Adult Vaccination: An Urgent Need to Prevent Hepatitis B in China. Hum. Vaccin. Immunother. 2016, 12, 773-778. [CrossRef]

6. Du, J.; Xu, Y.; Wang, J.; Liu, S.; Liu, Y.; Zhang, X.; Xu, E. 24 Year Outcomes of Hepatitis B Vaccination in Hangzhou, China. Hum. Vaccin. Immunother. 2015, 11, 2051-2060. [CrossRef]

7. Van Herck, K.; van Damme, P. Benefits of Early Hepatitis B Immunization Programs for Newborns and Infants. Pediatr. Infect. Dis. J. 2008, 27, 861-869. [CrossRef]

8. Poland, G.A.; Jacobson, R.M. Clinical Practice: Prevention of Hepatitis B with the Hepatitis B Vaccine. N. Engl. J. Med. 2004, 351, 2832-2838. [CrossRef]

9. Liu, R.; Li, Y.; Wangen, K.R.; Maitland, E.; Nicholas, S.; Wang, J. Analysis of Hepatitis B Vaccination Behavior and Vaccination Willingness among Migrant Workers from Rural China Based on Protection Motivation Theory. Hum. Vaccin. Immunother. 2016, 12, 1155-1163. [CrossRef] [PubMed]

10. Hu, Y.; Chen, Y.; Wang, Y.; Liang, H. Hepatitis B Vaccination among 1999-2017 Birth Cohorts in Zhejiang Province: The Determinants Associated with Infant Coverage. Int. J. Environ. Res. Public Health 2018, 15, 2915. [CrossRef] [PubMed]

11. Hennessey, K.; Schluter, W.W.; Wang, X.; Boualam, L.; Jee, Y.; Mendoza-Aldana, J.; Roesel, S.; Diorditsa, S.; Ehrenberg, J. Are We There Yet? Assessing Achievement of Vaccine-Preventable Disease Goals in Who's Western Pacific Region. Vaccine 2014, 32, 4259-4266. [CrossRef] [PubMed]

12. Li, S.; Huang, H.; Cai, Y.; Xu, G.; Huang, F.; Shen, X. Characteristics and Determinants of Sexual Behavior among Adolescents of Migrant Workers in Shangai (China). BMC Public Health 2009, 9, 195. [CrossRef] [PubMed]

13. National Bureau of Statistics. Migrant Workers Monitoring Investigation Report of China in 2018. 2019. Available online: http://www.stats.gov.cn/tjsj/zxfb/201904/t20190429_1662268.html (accessed on 3 August 2019). (In Chinese)

14. Zhu, C.; Geng, Q.; Yang, H.; Chen, L.; Fu, X.; Jiang, W. Quality of Life in China Rural-to-Urban Female Migrant Factory Workers: A before-and-after Study. Health Qual. Life Outcomes 2013, 11, 123. [CrossRef]

15. Bhattarai, M.; Baniya, J.B.; Aryal, N.; Shrestha, B.; Rauniyar, R.; Adhikari, A.; Koirala, P.; Oli, P.K.; Pandit, R.D.; Stein, D.A.; et al. Epidemiological Profile and Risk Factors for Acquiring Hbv and/or Hcv in Hiv-Infected Population Groups in Nepal. Biomed. Res. Int. 2018, 2018, 9241679. [CrossRef] [PubMed] 
16. Dai, W.; Gao, J.; Gong, J.; Xia, X.; Yang, H.; Shen, Y.; Gu, J.; Wang, T.; Liu, Y.; Zhou, J.; et al. Erratum To: Sexual Behavior of Migrant Workers in Shanghai, China. BMC Public Health 2016, 16, 1027. [CrossRef] [PubMed]

17. Ji, Z.; Wang, T.; Shao, Z.; Huang, D.; Wang, A.; Guo, Z.; Long, Y.; Zhang, L.; Su, H.; Zhang, Q.; et al. A Population-Based Study Examining Hepatitis B Virus Infection and Immunization Rates in Northwest China. PLoS ONE 2014, 9, e97474. [CrossRef]

18. Zhang, Y.B.; Chen, J.Q.; Hao, Y.T.; Chen, W.H.; Ma, Z.C.; Liao, Y.X.; Zhang, R.; Zhu, Q.L. Comparisons and Analysis of the Hepatitis B Infection Status of Transient Population and Registered Residents in Shenzhen City. Chin. J. Dis Control Prev. 2012, 16, 424-427. (In Chinese)

19. Action Plan for Prevention and Treatment of Viral Hepatitis in China (2017-2020). Chin. J. Virol. 2018, 8, 1-5. (In Chinese)

20. National Bureau of Statistics of the People's Repulic of China. China Statistical Yearbook 2018. National Bureau of Statistics of the People's Repulic of China, 2018. Available online: http://www.stats.gov.cn/tjsj/ndsj/ 2018/indexeh.htm (accessed on 19 August 2019).

21. Bureau of Statistics of Chongqing, China. Statistical Yearbook 2018. Bureau of Statistics of Chongqing, China, 2018. Available online: http:/tij.cq.gov.cn//tinj/2018/indexch.htm (accessed on 19 August 2019). (In Chinese)

22. Gong, T.; Gong, Y.; Deng, D.; Ye, M.L. A Cross-Sectional Study on Hepatitis B in Migrant Workers of Chongqing. Mod. Prev. Med. 2007, 20, 3842-3844. (In Chinese)

23. Health Commsssion of Chongqing, China. Statistic Yearbook 2016. Health Commsssion of Chongqing, China, 2017. Available online: http://wsjkw.cq.gov.cn/sjfx/ (accessed on 19 August 2019). (In Chinese)

24. Guirgis, M.; Yan, K.; Bu, Y.M.; Zekry, A. General Practitioners' Knowledge and Management of Viral Hepatitis in the Migrant Population. Intern. Med. J. 2012, 42, 497-504. [CrossRef]

25. Van der Veen, Y.J.; Voeten, H.A.; de Zwart, O.; Richardus, J.H. Awareness, Knowledge and Self-Reported Test Rates Regarding Hepatitis B in Turkish-Dutch: A Survey. BMC Public Health 2010, 10, 512. [CrossRef]

26. World Health Organization. Frequently Asked Questions on Hepatitis B Vaccination in China. 2014. Available online: https:/www.who.int/china/news/detail/18-08-2014-frequently-asked-questions-onhepatitis-b-vaccination-in-china (accessed on 19 August 2019).

27. Yan, H.; Yan, N. Analysis of the Factors Influencing the Active Acceptance of Hepatitis B Vaccine in Adults. J. Qiqihar Univ. Med. 2015, 36, 3063-3064. (In Chinese)

28. Liu, C.; Lvu, Y.K. Analysis on Hepatitis B Related Kap among Migrants in Beijing. Chin. J. Health Educ. 2015, 31, 1029-1032. (In Chinese)

29. Xie, Z.Y.; Yan, T.; Fei, Y.; Ying, J.H.; Li, Z.W.; Cao, G.W. Investigation of Hbv Infection and Hepatitis B Vaccination Coverage in Children of Migrant Workers in Pudong New Area, Shanghai, China. Acad. J. Second Mil. Med. Univ. 2016, 37, 1464-1469. (In Chinese)

30. Grabenstein, J.D. What the World's Religions Teach, Applied to Vaccines and Immune Globulins. Vaccine 2013, 31, 2011-2023. [CrossRef]

31. Rajamoorthy, Y.; Radam, A.; Taib, N.M.; Rahim, K.A.; Wagner, A.L.; Mudatsir, M.; Munusamy, S.; Harapan, H. The Relationship between Perceptions and Self-Paid Hepatitis B Vaccination: A Structural Equation Modeling Approach. PLoS ONE 2018, 13, e0208402. [CrossRef]

32. Cameron, D. The Inhibition of Behavior: Working Concepts. Am. J. Psychiatry 1951, 107, 701-705. [CrossRef]

33. Rajamoorthy, Y.; Radam, A.; Taib, N.M.; Rahim, K.A.; Munusamy, S.; Wagner, A.L.; Mudatsir, M.; Bazrbachi, A.; Harapan, H. Willingness to Pay for Hepatitis B Vaccination in Selangor, Malaysia: A Cross-Sectional Household Survey. PLoS ONE 2019, 14, e0215125. [CrossRef]

(C) 2019 by the authors. Licensee MDPI, Basel, Switzerland. This article is an open access article distributed under the terms and conditions of the Creative Commons Attribution (CC BY) license (http://creativecommons.org/licenses/by/4.0/). 